\title{
A ROCKET PAYLOAD USING FOCUSING X-RAY OPTICS FOR THE OBSERVATION OF SOFT COSMIC X-RAYS
}

\author{
P. GORENSTEIN, B. HARRIS, H. GURSKY, and R. GIACCONI
}

\begin{abstract}
A rocket payload utilizing focusing optics has been designed and constructed for the detection and localization of cosmic X-ray sources. The instrument is designed to be used in a scanning mode. The sensitivity of the instrument covers the wavelength bands $80 \AA$ to $44 \AA$ and $20 \AA$ to $10 \AA$ with a total field of view of $2^{\circ} \times 8^{\circ}$. A telescope operating on the principle of grazing incidence reflection instantaneously focuses the $\mathrm{X}$-rays from a distant source in one dimension upon one member of four proportional counter elements located in the focal plane. Location precision from a single scan is about $0.1^{\circ} \times 1^{\circ}$ for the stronger sources. The telescope consists of eight nested sheets of re-enforced glass whose reflecting surfaces are overcoated with an evaporated layer of chromium to improve reflection properties at the shorter wavelengths. The effective collecting area of the mirror assembly for on-axis radiation is about $160 \mathrm{~cm}^{2}$ at $80 \AA$, and $40 \mathrm{~cm}^{2}$ at $10 \AA$. The focal plane detector is a multiwire proportional counter with a one micron polypropylene entrance window and contains a supply of propane gas regulated to a constant pressure of $50 \mathrm{~cm}$. In the wavelength range $80 \AA-10 \AA$ the focusing technique results in (1) greater sensitivity, (2) better angular resolution, and (3) improved detector reliability compared to non-focusing techniques. (Published, Nuclear Instruments and Methods 91 (1971) 451.)
\end{abstract}

\section{DISCUSSION}

E. Trendelenburg: What is the advantage of the one dimensional focusing systems as compared with the parabolic mirror double focusing method?

$R$. Giacconi: The advantages of one dimensional focusing systems as compared to two dimensional are larger collecting area and larger field of view. These are important in a survey experiment when one wishes to observe a large region of the sky in a relatively short time. On the other hand, one dimensional systems do not have as good overall angular resolution, are more susceptible to source confusion, and are not as well suited to studying the angular structure of an extended source.

A. Scheepmaker: What is the best window material for proportional counter's available in respect to transmission properties?

R. Giacconi: I do not know of any 'best' material, for different experiments different filters can be used, their properties are reported in the literature. 